\title{
Iztmo: \\ A New and Intuitive Way to Connect People
}

\author{
Danielle Gandarillas, Gil Guigon, and Ilana Paterman \\ Escola Superior de Desenho Industrial, \\ Universidade do Estado do Rio de Janeiro, Brazil \\ http://www.esdi.uerj.br
}

\begin{abstract}
Iztmo is a conceptual project of an electronic percussion instrument that works under a network environment to establish communication among multiple users. A new language is then created, based on rhythm, and enriched by each person's cultural background with the main purpose of being in harmony. Iztmo emits up to five sounds, divided in different ways of interaction: the user can strike it, rattle it, and scrape it. Some might play well, some might have no rhythm. But everybody will learn sharing this experience. A self-standing video was made to explain the concept and to demonstrate how the experience works.
\end{abstract}

Keywords: Interaction design - tangible media - conceptual design digital device - communication - connection - music - experience.

\section{Introduction}

How to use new technologies to promote the exchange of cultural diversity, but preserving it, by making people from all over the world communicate through an intuitive, universal language? In one of the most instinctive ways of expression, we've come to an answer.

Iztmo is a device that promotes people with different backgrounds to become in harmony. Through the language of percussion rhythm, they can exchange their musical influences, teaching and learning, or even create codes to explore Iztmo's communication possibilities.

The process is simple: just turn your Iztmo on, and you are connected, by random, to up to five other users in the world. If the room you enter is too noisy, or too slow, you push a single button, and you'll be visiting other rooms which are being created in real time. These rooms, kindly called Izt-rooms, work like virtual drum circles, where everybody lets the rhythm run free, getting into a same pace.

\section{Interaction}

Like a percussion instrument, Iztmo is easy and intuitive to play. There are three actions you can do, in a total of five different sounds: striking (top, bottom, lateral), rattling and scraping. 


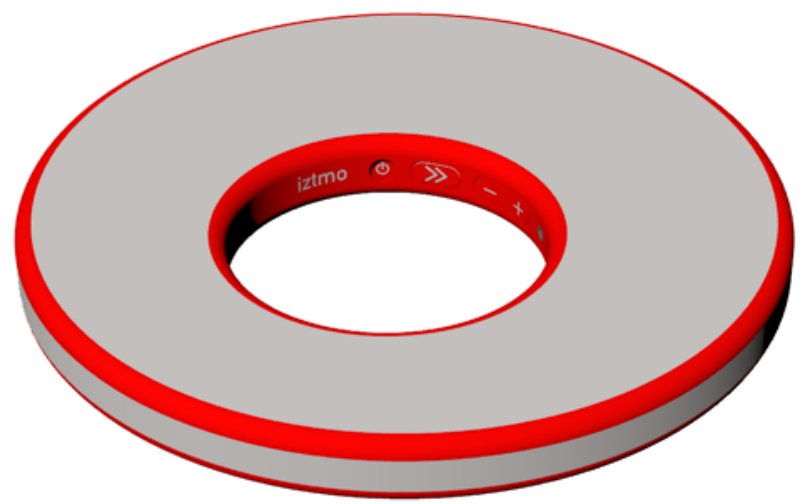

Fig. 1. Iztmo's device

Iztmo works along with a website, that enables functions not supported by the device: you can, for instance, configure the sounds of your device, and record and edit your musical experiences in a history of interactions. The decision to transfer additional features to a website, instead of increasing the device's functionality, is logic: we're dealing with an instrument, being necessary to give as much freedom as possible for the user. The device, then, has only the essential buttons to be connected and play.

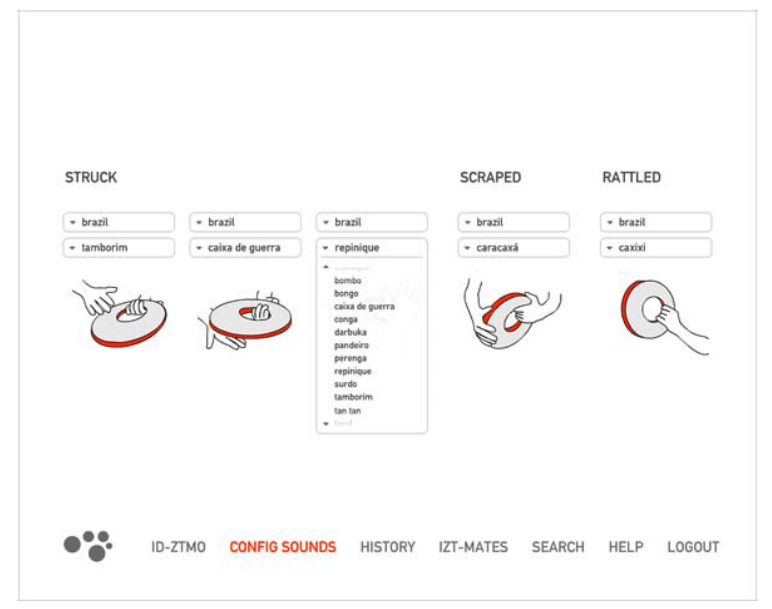

Fig. 2. Iztmo's website: the page to configure sounds

Since Iztmo introduces a new way to communicate, users need new identities. The ID-ztmo is a personal combination of sounds, i.e., a customized riff, that works as a name. You can be recognized in a room by playing your ID-ztmo; you can also be connected to a specific person by playing this person's ID-ztmo. 
Users you interact with are more than mere responses to the sounds you make. They are your Izt-mates. On the website, it's possible to add a user you've played with, by looking at the 'history' button; you can also visualize if this user's device is turned on. Then, you can be connected to this Izt-mate by a single click, and continue that great jam session from yesterday. Your Izt-mates can be managed in an user-friendly interface, which allows you to organize them as you want, besides checking out if they are online at the website, at the device, or both.

\section{Experience}

It was necessary to verify what kind of communication, if any, could be established through rhythm. To see how Iztmo would work, several usability tests were made; since it's a conceptual product, we had to find a way to simulate Iztmo's conceptual aspects without counting with a real prototype.

Volunteers were called to sit in chairs organized in a circle. But there is a detail on that: they couldn't see each other, since the chairs were turned to the circle's exterior. In front of each volunteer, there was a table and, over it, a pot lid, a fork, and a matchbox. They were asked to use these objects to create sounds, as well as the own table, in the way they wanted, in one single rule: not to have any visual contact with the others.

The result was more than satisfactory; some of the volunteers could easily find a rhythm in common; some couldn't, but, after a while, they were all playing together, improvising new sounds and suggesting changes. And, what is more important: they all enjoyed the experience.

\section{Conclusion}

Iztmo does not exist. As a conceptual project, its goal is to provoke alternative ways to develop communication media in a networked digital world. Besides, conceptual approaches lead us to re-think social values and everyday habits.

A new language, with no visual communication, would be created with Iztmo. Feelings could be easily interpreted by sounds: a strong hit for rage, for example. It is a level of communication quite different from the ones used in digital media, for it does not need any representation to be understood (such as words or images). There are several research projects in the area of collaborative music, but they differ from Iztmo in many aspects, like in the presence of a visual language [1, 3], 4, [5] and in the fact that users are at a same real place [1, [2, 4]. The real presence of users is, again, a form of visual communication. Besides, Iztmo is a portable device.

This project was developed for Microsoft Research Design Expo 2004, which had the theme Connecting People to People, from friends to strangers. Connecting people with different cultures through a universal language, in a way to promote harmony between them, is Iztmo's premise. During the process, we have found out that some public events promote people's connection through 
music: drum circles, for example. Such manifestations reveal the human need of being part of public rituals, especially in big cities.

There are different levels of human needs that an interaction designer should be aware of. The more we realize which are these different levels, the better digital technologies will be applied to innovative, richer experiences.

\section{References}

1. Blaine, T., Perkis, T.: The Jam-O-Drum interactive music system: a study in interaction design. In: Proceedings of the conference on Designing interactive systems (DIS '00), ACM Press, New York (2000)

2. Oshima, C., Nishimoto, K., Suzuki, M.: Family ensemble: a collaborative musical edutainment system for children and parents. In: Proceedings of the 12th annual ACM international conference on Multimedia, New York, NY, USA (2004)

3. Gurevich, M.: JamSpace: designing a collaborative networked music space for novices. In: Proceedings of the 2006 conference on New interfaces for musical expression, Paris, France (2006)

4. Patten, J., Recht, B., Ishii, H.: Interaction techniques for musical performance with tabletop tangible interfaces. In: Proceedings of the 2006 ACM SIGCHI international conference on Advances in computer entertainment technology, Hollywood, California (2006)

5. Mueller, F.F., Agamanolis, S.: Pervasive gaming: Sports over a distance. Computers in Entertainment (CIE) 3(3) (2005) 